\title{
Spinning Q-balls in Five-Dimensional Spacetime
}

\author{
Bintoro A. Subagyo \\ Departement of physics, Faculty of Science, \\ Institut Teknologi Sepuluh Nopember \\ Kampus ITS Sukolilo, Surabaya 60111
}

\begin{abstract}
We investigate $q$-balls in the $\mathrm{d}=5$ dimensional Minkowski spacetime background with axially symmetric ansatz. In this case, q-balls have non-zero angular momentum for spinning problems. We construct spinning q-balls in five dimensions and solve the solutions of second order elliptic partial differential equations both using analytical and numerical approach since no closed form of the equations following by appropriate boundary conditions. This work only considers the case with equal angular momentum rather than two different angular momenta. Passing the solutions, we discuss the global charge and their properties compared to Coleman q-balls. The solutions exist in some range of frequencies. The main features of q-balls are still recovered in this construction.
\end{abstract}

Keywords: solitons, q-balls, axially symmetric.

*Corresponding author: b_anang@physics.its.ac.id

http://dx.doi.org/10.12962/j24604682.v15i2.5254

2460-4682 (C)Departemen Fisika FSains-ITS

\section{INTRODUCTION}

The solitons entered the realm of physics in a very wide range from a wave traveling down Edinburghs Union Canal to condensed matter and cosmology as well [1-3]. In high energy physics, soliton can be regarded as an important ingredient in some models [4]. In this work, solitons refer to any particle-like solution of non-linear field theory. Such configurations are asymptotically flat and globally stationary, although not necessarily stable, their energy is localized in a finite region of space.

The solitons can be classified into two general types: topological solitons and non-topological solitons. The magnetic monopole solution of $t$ Hooft [5] and Polyakov [6] is the bestknown example of the topological soliton. Their stability is ensured by the presence of a conserved topological charge. On the other hand, the non-topological solitons do not possess a conserved topological and thus might be unstable. The first systematic study of non-topological solitons has been worked out in the 70s by Freidberg, Lee, and Sirlin [7] in the context of the quantum chromodynamics hadron (quark bag) model. In this context, they also performed the study of particle-like solutions in an effective model with a complex non-linear scalar field. Several years later, the terminology of Q-balls was proposed by Coleman in 1985 [8] to describe such solutions. Their main feature is that the complex scalar field possesses a harmonic time dependence. These solutions also exist for a certain range of frequencies, some of the configurations being stable.

The studies of q-balls in four-dimensional spacetime, as well as their spinning generalization, have been made in many references. When q-balls coupled to gravity, boson stars arise.
Their solutions are also localized with finite energy. The spinning counterpart of boson stars was studied by Yoshida [8] and continued systematically in [9]. In curved spacetime, boson stars show the same behavior approaching the maximum of frequency and show the inspiraling pattern as approaching their minimal value of frequency. The q-balls and boson stars in the anti de-Sitter (AdS) spacetime background was also studied with and without self-interaction potential [10].

The main purpose of this article is to study the generalization and also the existence of spinning q-balls in fivedimensional Minkowski spacetime. The solutions are solved numerically of a set partial differential equations. In section II we construct the action and the field equations by taking standard variation to the action. In order to get axially symmetric solutions, we employ suitable ansatz and boundary conditions. Thus, we can calculate the global charges. In section III we discuss the solutions in five dimension as we also mention the solutions in four dimension. In section IV, we present our results on spinning cases for the field and also their global charges. In the last section, we discuss some further remark as well in the present of gravity coupled to the field.

\section{THE MODEL}

\section{A. Action and the field equation}

In order to construct q-balls, we consider a complex scalar field, $\Phi$, on five-dimensional spacetime background described 
as

$$
\begin{aligned}
S & =\int d^{D} x \sqrt{-g} L_{M} \\
& =\int d^{5} x \sqrt{-g}\left[-\frac{1}{2} g^{\mu \nu}\left(\Phi_{, \mu}^{*} \Phi_{, \nu}-\Phi_{, \nu}^{*} \Phi_{, \mu}\right)\right]
\end{aligned}
$$

where $g$ is metric determinant, asterisk mark $\left(^{*}\right)$ denote complex conjugate of the scalar field, $\Phi_{, \mu}=\partial \Phi / \partial x^{\mu}$, and $U(|\Phi|)$ is the scalar field potential. Lagrangian in the action (1) contains the usual kinetic term and the potential term.

In order to get non-topological solutions, q-balls, the potential has to obey special conditions, the non-linear potential. One of the potentials which fulfill our conditions is the nonrenormalizable potential [7],

$$
U(|\Phi|)=\lambda|\Phi|^{2}\left(|\Phi|^{4}-a|\Phi|^{2}+b\right) .
$$

The parameters $\lambda, a$, and $b$ in this work are picked as 1,2 and 1.1 respectively following the work of $[7,9,10]$. The potential has a minimum $U(|\Phi|)=0$ at $\Phi=0$ and in other finite value of $\Phi$ determined by the parameters of potential. If we take the square term of potential as $U(|\Phi|)=m|\Phi|^{2}$, then the boson mass is $m=\sqrt{\lambda b}$. The boson mass depend on the coefficient of square term of potential.

Lagrangian density in the action (1) is invariant to global phase transformation $\Phi \rightarrow \Phi e^{i \alpha}$ resulting in conserved current density

$$
j^{\mu}=-i\left(\Phi^{*} \Phi_{, \mu}-\Phi \Phi_{, \mu}^{*}\right), \quad j_{\mu}^{\mu}=0 .
$$

The total charge of q-balls can be obtained from the integration of current density, $j^{0}$. Variation of action (1) respect to scalar field yield the equation of motion,

$$
\frac{1}{\sqrt{-g}} \partial_{\mu}\left(\sqrt{-g} \partial^{\mu} \Phi\right)=\frac{\partial U}{\partial|\Phi|^{2}} \Phi .
$$

The equation (4) is the Klein-Gordon equation for the scalar field as usual. The energy-momentum tensor $T_{\mu \nu}$ can be obtained from

$$
\begin{aligned}
T_{\mu \nu}= & g_{\mu \nu} L_{M}-2 \frac{\partial L_{M}}{\partial g^{\mu \nu}} \\
= & \left(\Phi_{, \mu}^{*} \Phi_{, \nu}+\Phi_{, \nu}^{*} \Phi_{, \mu}\right)- \\
& g_{\mu \nu}\left[\frac{1}{2} g^{\alpha \beta}\left(\Phi_{, \alpha}^{*} \Phi_{, \beta}+\Phi_{, \beta}^{*} \Phi_{, \alpha}\right)+U(|\Phi|)\right]
\end{aligned}
$$

In this work, we solved equation (4) and their corresponding energy of equation (5) numerically.

\section{B. Ansatz}

In five dimension, we have two directions of angular momenta. However, in this work, we consider only a single angular momentum and set to vanish one of the other angular momenta. We are interested in constructing the stationary and axially symmetric solutions of q-balls with an azimuthal isotropic coordinate. Thus, the geometry of spacetime admits two-Killing vector fields. They are asymptotically timelike Killing vector fields, $\partial_{t}$, and spacelike Killing vector fields, $\partial_{\varphi}$. In term of spherical coordinate $(t, r, \theta, \psi, \varphi)$, a line element of five-dimensional Minkowski spacetime can be written as

$$
\begin{aligned}
d s_{5}^{2}= & -d t^{2}+d r^{2}+r^{2} d \theta^{2}+r^{2} \cos ^{2} \theta d \psi^{2}+ \\
& r^{2} \sin ^{2} \theta d \varphi^{2}
\end{aligned}
$$

In (6), all of the metric functions depend on $r$ and $\theta$ only. For the scalar field, we use the stationary ansatz

$$
\Phi(t, r, \theta, \psi, \varphi)=\phi(r, \theta) e^{i(n \varphi-\omega t)},
$$

where $\phi(r, \theta)$ is a real function, $\omega$ and $n$ are real constants. The requirement of single-valuedness of the scalar field needs that $\Phi(\varphi)=\Phi(2 \pi+\varphi)$. Consequently, $n$ must be an integer and known as an azimuthal winding number. Together with the axially symmetric ansatz (7) and Lagrangian, one shell get a partial differential equation to be solved in order to obtain the q-balls solutions. When $n=0$ and the scalar field depend on $r$ only, then the ansatz will approach the stationary spherical symmetric limit.

\section{The boundary conditions}

To find the solutions, we consider that q-balls must be globally regular and they have finite energy and energy density as well. Our solutions have to meet boundary conditions in conjunction with equation (4). For rotating axially symmetric q-balls, boundary conditions must be specified both at the origin, at infinity, on the $\theta=0$, and, using the reflection symmetry in the $\theta=\pi / 2$ plane. Power series analysis around origin, $r=0$, pointed out the following boundary conditions which are also true for spherically symmetric

$$
\Phi \|_{r=0}=0 .
$$

At the infinity, the scalar fields have to vanish

$$
\Phi \|_{r \rightarrow \infty}=0 .
$$

For $\theta=0, \pi$ we employ the boundary conditions

$$
\Phi \|_{\theta=0, \pi}=0,
$$

and for equatorial plane solutions

$$
\Phi \|_{\theta=\pi / 2}=0
$$

\section{The global charges}

The energy and angular momentum of q-balls can be obtained directly from the energy-momentum tensor. The expression for the mass-energy $E$ and angular momentum $J$ of the configurations given by

$$
E=\int T_{t t} d^{4} x
$$


and

$$
J=\int T_{t \varphi} d^{4} x
$$

The conserved scalar charge $Q$ is obtained from the timecomponent of the current

$$
Q=-\int J^{t} d^{4} x
$$

In the equations (12-14), integrations work over space volume. The charge and angular momentum are not independent quantities. From equations (13) and (14), one can obtain the quantization relation for the angular momentum

$$
J=n Q
$$

The relation (15) derived first in [10]. Thus a spherically symmetric solution with has no angular momentum, .

\section{THE SOLUTIONS}

We shall emphasize that our solutions are focusing only with rotation on a single plane instead of two planes, implies that $T_{t \varphi} \neq 0$. Before entering into five-dimensional cases, we shall mention some basic results of four-dimensional q-balls.

\section{A. Spherically symmetric solutions}

In the static model solutions, the ansatz (7) have to take $n=0$ and the scalar field depend on $r$ only, $\varphi(r)$. In this limit, the mass-energy of spinning q-balls given by

$$
\begin{aligned}
E & =\int_{0}^{\infty} T_{t t} d^{4} x \\
& =2 \pi^{2} \int_{0}^{\infty} r^{3}\left[\omega^{2} \phi^{2}+\left(\partial_{r} \phi\right)^{2}+U(|\phi|)\right] d r
\end{aligned}
$$

and their charge

$$
Q=4 \pi^{2} \omega \int_{0}^{\infty} \phi^{2} r^{3} d r
$$

The fundamental solutions of static q-balls also behave like their four-dimensional counterparts. The mass-energy and charge are going to diverge approaching the limiting value of maximum and minimum frequencies. The scalar field $\phi$ and energy density $T_{t t}$ localized.

The main features of four dimensional q-balls show that the energy-mass increased as the frequency goes to maximum and minimum bound. Thus, the q-balls solutions only exist between the range of frequency.

\section{B. Spinning q-balls in five dimension}

The axially spinning solutions are obtained when we consider in the case of $n \neq 0$ and the scalar field depend on $r$ and $\theta$. In five dimensional spherical coordinate, the scalar field equations (4) take the form

$$
\partial_{r r} \phi+\frac{1}{r^{2}} \partial_{\theta \theta} \phi+\left(\frac{1}{r^{2} \sin \theta \cos \theta}-\frac{2}{r^{2}} \tan \theta\right) \partial_{\theta} \phi+\left(\omega^{2}-\frac{n^{2}}{r^{2} \sin ^{2} \theta}\right) \phi-\frac{d U}{d \phi}=0 .
$$

The mass-energy of spinning q-balls have additional term compared to spherically symmetric solutions,

$$
E=\int_{0}^{\infty} T_{t t} d^{4} x=4 \pi^{2} \int_{0}^{\infty}\left[\left(\partial_{r} \phi\right)^{2}+\frac{1}{r^{2}} \phi \omega^{2} \phi^{2}+\left(\partial_{r} \phi\right)^{2}+U(|\phi|)\right] r^{3} \sin ^{2} \theta d r d \theta
$$

For rotating Q-balls, the charge and range of frequency are not changed. These features are also found in four dimensional cases [7-9], which are determined only by their shape of the potentials. The spinning q-balls also exist in the frequency range of $\omega_{\text {min }}^{2}<\omega^{2}<\omega_{\text {max }}^{2}$, where

$$
\omega_{\min }^{2}=\lambda\left(b-\frac{a^{2}}{4}\right) \text {. }
$$

and the maximal value of frequency given by the boson mass,

$$
\omega_{\max }^{2}=\lambda b=m^{2} .
$$

\section{THE RESULTS}

In this work, we present the simplest non-topological solitons solutions, q-balls, even in five dimensional spacetime background. The solutions are found by combining both using a numerical approach. For simplicity, we consider only the fundamental q-balls without nodes. The model in this work provides the simplest non-topological soliton even in five dimensions. The approaches and numerical techniques for solving the problems based on solving the elliptic differential equation(s). 


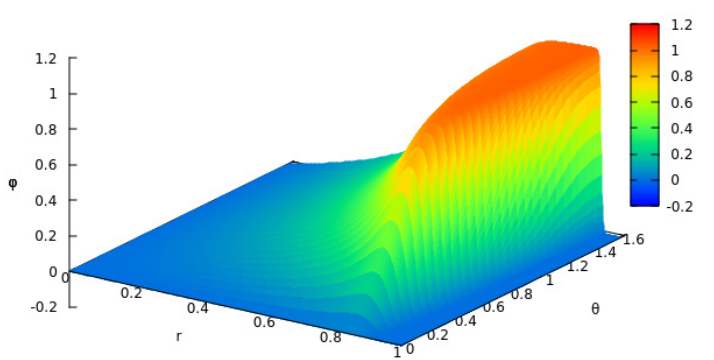

(a)

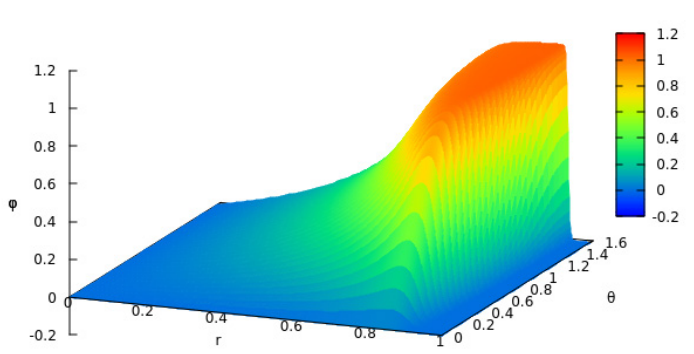

(c)

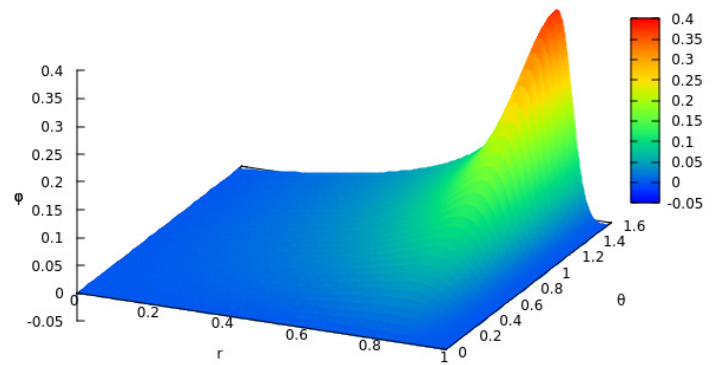

(b)

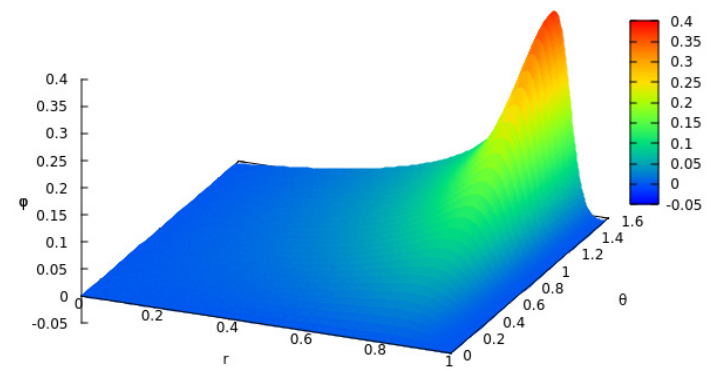

(d)

FIG. 1: (color online) The q-balls solutions for $\omega=0.5$, (a) for $n=1$ and (b) for $n=2$ while the q-balls solutions with $\omega=1.048$, (c) for $n=1$ and (d) for $n=2$

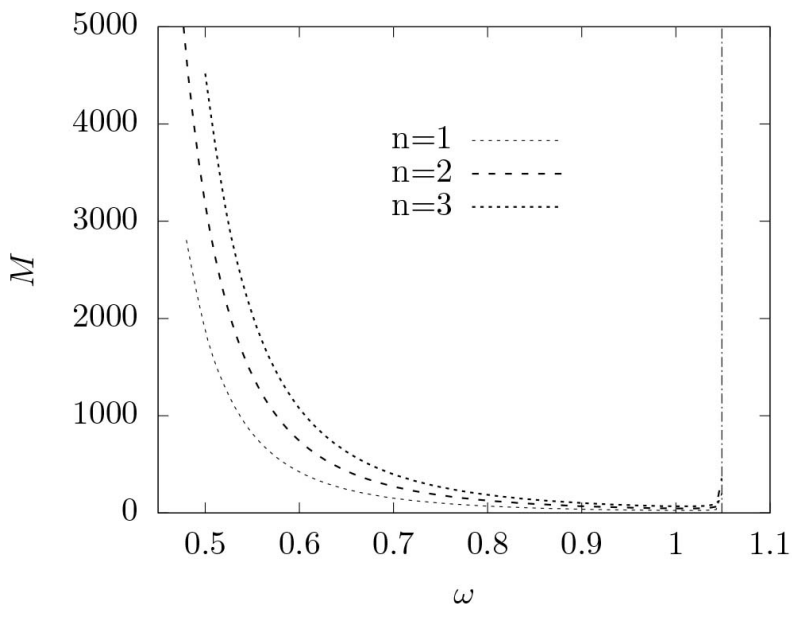

FIG. 2: The mass-energy (M) of q-balls are shown as a function of the frequency for several values of winding number, $n$

The stationary axially symmetric ansatz is applied on the field equations and solve them numerically. We solved nonlinear elliptic partial differential equations subject to boundary conditions. The equations are solved in the grid system with $251 \times 30$ points. Integration is covering the region $0 \leq \bar{r} \leq 1$ and $0 \leq \theta \leq 2 \pi$. We employ radial compactified coordinate, $\bar{r}=r /(1+r)$, which maps radial infinity into $\bar{r}=1$. The numerical calculations are based on the Newton-Raphson method and are carried out with the help of the FIDISOL package $[11,12]$.

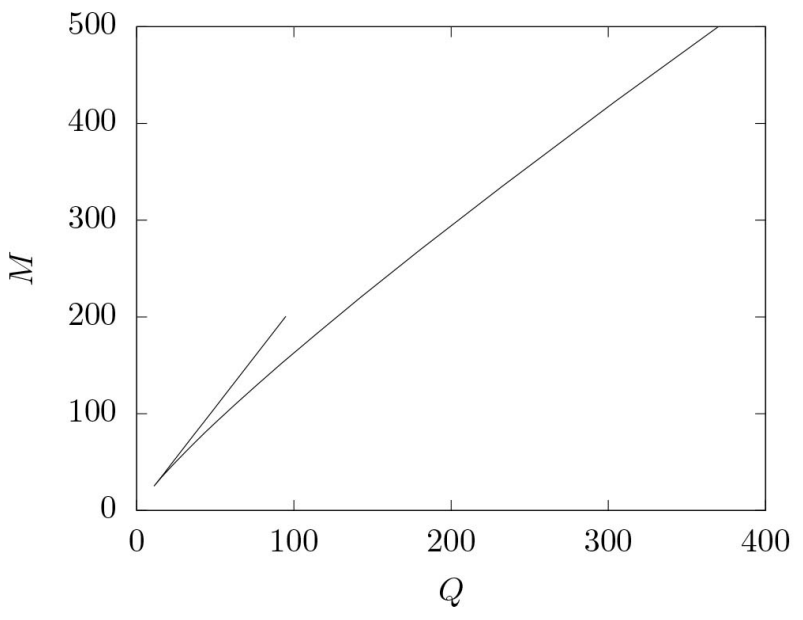

FIG. 3: The mass-energy (M) and Noether charge (Q) of q-balls are plotted

The rotating generalization of q-balls in five dimensions is obtained by taking the value of in the field ansatz. The solutions at the maximum and minimum value of frequency show the generic features, both static and spinning q-balls, of fourdimensional one. The mass-energy and angular momentum approach infinity toward their limiting frequencies as shown in Fig. 1. The field solutions, picture in figure 1, exhibit localized rotating soliton solution. The peak of the solutions turn to narrow when increasing the frequency. The solutions are exist in certain range of frequency as figured out in Fig. 2 Approaching the limiting value of frequency, numerics also 
become more tricky since in this limit they go to diverge.

The mass-energy $M$ and charge of rotating q-balls in five dimension exhibit in Fig. 3. The relation of global charge on frequency are analogous between five and four dimensional rotating q-balls. We shall mention also stability of the solutions. The situations of rotating q-balls stability can be read off from Fig. 3. One can see two branches of solutions. The lower branch of the solutions are classically stable. The lower branch ends at the critical point occur at the mass and charge approaching minimal values. Otherwise, the upper branch start from this critical point.

\section{FURTHER REMARKS}

In this work, we present the solution of axially spinning qballs in five dimensional spacetime. This study was motivated by development of field theory in higher spacetime dimension. The results show that many feature of their four dimensional counter part also be exhibited in this work. All solutions have upper and lower bound frequencies which is determined by scalar field mass and also the constant in the potentials.

This solutions can be expanded in some directions. One can study a straightforward generalization in more higher dimension. We expect most of the features will be covered in this approach. When gravity are coupled to the scalar field, we have boson stars that have different behaviour as the gravity contribute the non-linearity to the theory.

In the end, we shall say that the study of Q-balls and their gratating counterpart is interesting from yet many point of view. This work also ideal cases for investigating simple various numerical approach on solving axially symmetric problems, which can be applied into more complex models.

\section{Acknowledgments}

B. S. thank Doni L. Anggara for collaboration in some stages of this work. We gratefully acknowledge partial support by the Institut Teknologi Sepuluh Nopember local grant.

\section{REFERENSI}

[1] P.G. Drazin, R. S. Johnson, "Solitons: an introduction", Cambridge University Press, 1989.

[2] G.'t Hooft, "Magnetic Monopoles in Unified Gauge Theories", Nucl. Phys. B, vol. 79, pp. 276, 1974.

[3] A.M. Polyakov, "Particle Spectrum in the Quantum Field Theory", JETP Lett. vol. 20, pp. 194, 1974. [Pisma Zh. Eksp. Teor. Fiz. vol. 20, pp. 430, 1974].

[4] S.R. Coleman, "Q Balls", Nucl. Phys. B, vol. 262, pp. 263, 1985 [Erratum-ibid. B, vol. 269, pp. 744, 1986].

[5] R. Friedberg, T. D. Lee and A. Sirlin, "A Class of Scalar-Field Soliton Solutions in Three Space Dimensions", Phys. Rev. D, vol. 13, pp. 2739, 1976.

[6] M.S. Volkov and E. Wohnert, "Spinning Q balls", Phys. Rev. D, vol. 66, pp. 085003, 2002 [hep-th/0205157].

[7] E. Radu and M. S. Volkov, "Existence of stationary, non- radiating ring solitons in field theory: knots and vortons", Phys. Rept. vol. 468, pp. 101, 2008 [arXiv:0804.1357 [hep-th]].

[8] S. Yoshida and Y. Eriguchi, "Rotating boson stars in general relativity", Phys. Rev. D, vol. 56, pp. 762, 1997.

[9] B. Kleihaus, J. Kunz and M. List, "Rotating boson stars and Qballs", Phys. Rev. D, vol.72, pp. 064002, 2005 [gr-qc/0505143].

[10] E. Radu and B. Subagyo, "Spinning scalar solitons in antide Sitter spacetime", Phys. Lett. B, vol. 717, pp. 450, 2012 doi:10.1016/j.physletb.2012.09.050 [arXiv:1207.3715 [gr-qc]].

[11] W. Schönauer and R. Weiß, J. Comput. Appl. Math., vol. 27, pp. 279, 1989.

[12] M. Schauder, R. Weiß and W. Schönauer, The CADSOL Program Package, Universität Karlsruhe, Interner Bericht Nr. 46/92 (1992). 